\title{
Epidemiological and clinical profile of adult patients with Blastocystis sp. infection in Barcelona, Spain
}

Fernando Salvador ${ }^{1 *}$, Elena Sulleiro², Adrián Sánchez-Montalvá ${ }^{1}$, Carmen Alonso 3,4, Javier Santos,4, Isabel Fuentes ${ }^{5}$ and Israel Molina ${ }^{1}$

\begin{abstract}
Background: Blastocystis spp. are among the most frequently observed intestinal parasites in humans. Despite the discovery of Blastocystis approximately 100 years ago, limited information is available regarding its pathogenesis, genetic diversity, and available treatment options. The aim of this study was to describe the epidemiological and clinical characteristics of patients with Blastocystis sp. infections diagnosed at Vall d'Hebron University Hospital (Barcelona, Spain).
\end{abstract}

Methods: A retrospective observational study was performed which included all adult patients who attended Vall d'Hebron University Hospital from February 2009 to March 2014 that had Blastocystis sp. detected in their stool.

Results: Four hundred eighteen patients were included, the median age was 36 (18-86) years and 236 (56.5 \%) were men. Regarding patient symptoms, 234 (56\%) patients were completely asymptomatic, 92 (22\%) patients had symptoms, and 92 (22\%) patients had symptoms that could be attributed to other causes. Of the 92 patients with symptoms not attributable to other etiologies except for Blastocystis infection, the most frequent symptoms were diarrhea (61 patients, $66.3 \%$ ) and abdominal pain (34 patients, $37 \%$ ). Additionally, nine (9.8 \%) patients had cutaneous manifestations. Thirty-one (7.4\%) patients received specific treatment for Blastocystis infection. The clinical response of treated patients was varied. Five patients experienced complete resolution of symptoms, 12 patients reported improvement of clinical symptoms, eight patients described no clinical improvement, and information was unavailable for six patients.

Conclusions: Blastocystis infection was detected in 418 patients, most of them foreign-born. Although the vast majority of patients were asymptomatic, $22 \%$ of patients had gastrointestinal symptoms or cutaneous manifestations in the absence of other causes. Despite the scarce information available, given the safety of antiparasitic treatment, and the percentage of patients who experienced resolution or improvement of symptoms, treatment should be considered in patients with chronic symptoms.

Keywords: Blastocystis, Intestinal protozoa, Gastrointestinal symptoms, Metronidazole

\footnotetext{
* Correspondence: fmsalvad@vhebron.net

${ }^{1}$ Department of Infectious Diseases, Vall d'Hebron University Hospital,

Universitat Autònoma de Barcelona, PROSICS Barcelona, Barcelona, Spain

Full list of author information is available at the end of the article
}

\section{Ciomed Central}

(c) 2016 The Author(s). Open Access This article is distributed under the terms of the Creative Commons Attribution 4.0 International License (http://creativecommons.org/licenses/by/4.0/), which permits unrestricted use, distribution, and reproduction in any medium, provided you give appropriate credit to the original author(s) and the source, provide a link to the Creative Commons license, and indicate if changes were made. The Creative Commons Public Domain Dedication waiver (http://creativecommons.org/publicdomain/zero/1.0/) applies to the data made available in this article, unless otherwise stated. 


\section{Background}

Parasitic diseases are amongst the most important causes of morbidity and mortality worldwide, and it is estimated that nearly 340 parasite species infect humans. Of these species intestinal protozoa are the most prevalent [1]. The pathogenicity of several intestinal protozoa is well established, and their presence is usually associated with diarrhea and other intestinal manifestations (viz. Giardia duodenalis, Entamoeba histolytica, Cryptosporidium spp., Cystoisospora belli). Some protozoa cause significant morbidity and sometimes mortality, especially among immunosuppressed populations [2].

In contrast, the role of other intestinal protozoa in the development of human disease is not well established, and their presence in both symptomatic and asymptomatic patients is difficult to explain [1]. Blastocystis spp. are among the most frequently observed intestinal parasites in humans. Despite its description approximately 100 years ago, little information is available regarding their pathogenesis, genetic diversity, host range and available treatment options [3]. Blastocystis has a worldwide distribution, with higher prevalences reported in developing countries. The parasite is transmitted by the fecal-oral route, and animal reservoirs include chickens, rats and pigs [4]. Seventeen subtypes (STs) have been described based on analyses of the small subunit rRNA (SSU rRNA) gene. Only STs 1-9 have been found in humans, ST-3 being the most prevalent in epidemiological studies $[5,6]$.

Clinical manifestations associated with Blastocystis spp. carriage include diarrhea, abdominal pain, vomiting and other gastrointestinal symptoms. Cutaneous manifestations (urticaria) may also be associated with the presence of Blastocystis spp. [7]. Nevertheless, some investigators report no association between the presence of clinical manifestations and Blastocystis infection [8]. Hence, more information is required to properly assess the potential pathogenicity of this parasite.

Few studies have investigated the prevalence of Blastocystis infection in Spain, and most of them focused on children or symptomatic patients [9-11]. The aim of the present study was to describe the epidemiological and clinical characteristics of adult patients with Blastocystis infection and evaluate possible risk factors associated with the presence of clinical manifestations.

\section{Methods}

A retrospective observational study was performed at the Vall d'Hebron University Hospital, a tertiary hospital included in the International Health Program of the Catalan Health Institute (PROSICS Barcelona, Spain). The study included all adult patients ( $\geq 18$ years old) who attended the hospital from February 2009 to March 2014, had Blastocystis spp. in their stool and medical records available.
To establish the study cohort, a list of patients infected with Blastocystis sp. was compiled by examination of the Microbiology Department registry. Next, medical records were reviewed and for those patients with a medical record available, the following data was collected: epidemiological data (age, gender, country of origin, previous travels), immune status, clinical symptoms that could be related with Blastocystis sp. infection (gastrointestinal and cutaneous), detection of other intestinal parasites, treatment received and follow-up after treatment. Blastocystis sp. detection was performed by microscopic examination of stool samples following concentration using the Ritchie's formalin-ether technique.

According to the geographical origin of patients and recent travels, patients were classified into four epidemiological risk groups: immigrants, travelers (patients born in Spain who have traveled outside the country in the last year before their Blastocystis sp. infection), "visiting friends and relatives" (VFR, immigrants living in Spain who have traveled to their country of origin in the last year before their Blastocystis sp. infection), and autochthonous infections (patients born in Spain with no history of recent travel outside the country).

To identify risk factors associated with the presence of symptoms, patients were classified into three groups: asymptomatic patients, symptomatic patients, and patients with symptoms that could be attributable to causes other than Blastocystis sp. infection.

In patients who received specific treatment for their Blastocystis sp. infection, microbiological and clinical follow-up information was collected. Regarding clinical response to therapy, patients were classified into three groups: those who experienced complete resolution of symptoms, those who reported an improvement in their symptoms but not complete resolution, and patients who experienced no clinical improvement following treatment.

Categorical data are presented as absolute numbers and proportions, and continuous variables are expressed as medians and ranges. The $\chi^{2}$ test or Fisher's exact test, when appropriate, was used to compare the distribution of categorical variables, and the Mann-Whitney U-test for continuous variables. To evaluate the seasonality of the infection, the two-sample test of proportions was performed between seasons. Results were considered statistically significant if the 2 -tailed $P$-value was $<0.05$. SPSS software for Windows (Version 19.0; SPSS Inc, Chicago, IL, USA) was used for statistical analyses.

\section{Results}

After consulting the Microbiology Department registry, 510 possible cases of Blastocystis sp. infection were identified. After discarding patients less than 18 years old, duplicated patients, and patients lacking complete 
medical records, 418 cases remained for inclusion in the study (see Fig. 1). The epidemiological characteristics of patients are summarized in Table 1 . The median age of patients was $36(18-86)$ years, and $236(56.5 \%)$ were men. Regarding immigration status, 263 (62.9\%) patients were immigrants with median time of residence in Spain of 60 (1-480) months, $26(6.2 \%)$ patients were travelers, 10 (2.4\%) patients were VFR, and 119 (28.5\%) cases were autochthonous. Most patients were diagnosed in spring, although this difference was not statistically significant when compared to Blastocystis sp. infections diagnosed in winter (32.3 vs $22.2 \%, Z=1.66, P=0.08$ ), summer (32.3 vs $22 \%, Z=1.69, P=0.09)$, and autumn (32.3 vs $23.4 \%, Z=1.48, P=0.13$ ).

Sixty-six (15.8 \%) patients were immunocompromised. Forty-three patients had HIV infection, with a median of CD4+ cell count of $428(100-1,053)$ cells $/ \mathrm{mm}^{3}$ at the time their Blastocystis sp. infection was diagnosed, 11 patients had a solid tumor or a haematological malignancy, and 12 patients had rheumatological, gastrointestinal or dermatological diseases requiring immunosuppressive treatment.

Regarding patient symptoms, 234 (56 \%) patients were completely asymptomatic, $92(22 \%)$ patients had symptoms, and $92(22 \%)$ patients had symptoms that could be attributed to other causes. Information on the clinical characteristics of this cohort is available in Table 2 . Among patients with other etiologies that could be responsible for their symptoms, ten had been diagnosed with intestinal neoplasm, 24 patients with other infectious diarrhea (Clostridium difficile, Campylobacter jejuni, Salmonella enteritidis, Shigella disenteriae, Giardia duodenalis), six patients with inflammatory bowel disease, and 11 patients with irritable bowel syndrome. Other intestinal parasites found in the study population are described in Table 3. From the 92 patients with symptoms not attributable to etiologies other than Blastocystis sp. infection, the duration of symptoms was $<1$ month in $32(34.8 \%)$ patients, and $>1$ month in $60(65.2 \%)$ patients. The most frequent symptoms were diarrhea (61 patients, $66.3 \%$ ) and abdominal pain (34 patients, $37 \%)$, and $9(9.8 \%)$ patients had cutaneous manifestations including eczematous or urticarial dermatitis.

When comparing the clinical and epidemiological characteristics of the asymptomatic and symptomatic patients with symptoms attributable to Blastocystis sp. alone, asymptomatic patients were younger (median age 34 years $v s 42.5$ years, $Z=-3.896, P<0.0001$ ), included a larger proportion of migrants (89.7 vs $32.6 \%, X^{2}=$ $110.998, d f=1, P<0.0001)$, and included a greater proportion of immunocompromised patients (20.1 vs $5.4 \%$, $\left.\chi^{2}=10.573, d f=1, P=0.001\right)$ (Table 4).

Overall, 31 (7.4 \%) symptomatic patients received specific treatment for Blastocystis sp. infection: 27 patients received $750 \mathrm{mg}$ metronidazole three times a day for 10 days, two received $800 \mathrm{mg} / 160 \mathrm{mg}$ cotrimoxazole two times a day for 7 days, and two patients received sequential treatment with metronidazole and cotrimoxazole (10 days of $750 \mathrm{mg}$ metronidazole followed by 7 days of $800 \mathrm{mg} / 160 \mathrm{mg}$ cotrimoxazole). The clinical response

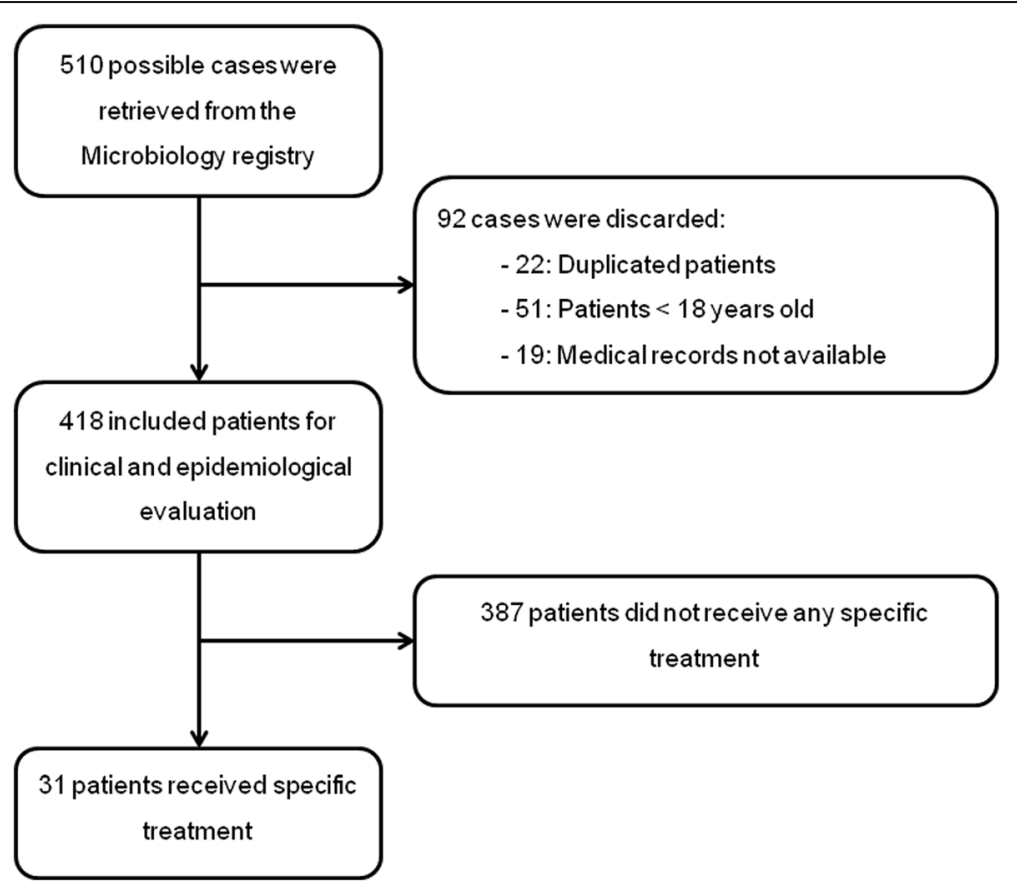

Fig. 1 Flow diagram depicting study cohort size and selection 
Table 1 Epidemiological characteristics of patients with Blastocystis sp. infection attended in Vall d'Hebron University Hospital, Barcelona (2009-2014). Data are reported as number (\%) of patients or median (range)

\begin{tabular}{ll}
\hline Characteristics & Number of patients $(n=418)$ \\
\hline Age, years & $36(18-86)$ \\
Gender, male & $236(56.5 \%)$ \\
Origin of patients & \\
$\quad$ Spain & $143(34.2 \%)$ \\
Latin America & $149(35.6 \%)$ \\
Sub-Saharan Africa & $98(23.4 \%)$ \\
Asia & $14(3.3 \%)$ \\
North Africa & $11(2.6 \%)$ \\
Other European countries & $3(0.7 \%)$ \\
Epidemiological risk group & \\
Immigrants & $263(62.9 \%)$ \\
Autochthonous & $119(28.5 \%)$ \\
Travelers & $26(6.2 \%)$ \\
Visiting friends and relatives & $10(2.4 \%)$ \\
\hline
\end{tabular}

Table 2 Clinical characteristics of patients with Blastocystis sp. infection attended in Vall d'Hebron University Hospital, Barcelona (2009-2014)

\begin{tabular}{ll}
\hline Characteristics & $\begin{array}{l}\text { Number of patients (\%) } \\
(n=418)\end{array}$ \\
\hline Presence of immunosuppression & $66(15.8)$ \\
HIV infection & $43(10.3)$ \\
Oncohaematological disease & $11(2.6)$ \\
Rheumatological disease & $6(1.4)$ \\
Other immunosuppressant conditions & $6(1.4)$ \\
Clinical groups according presence of symptoms \\
Asymptomatic patients & $234(56)$ \\
Symptomatic patients & $92(22)$ \\
Symptomatic patients with other & $92(22)$ \\
possible explanation & \\
Symptoms among symptomatic patients $(n=92)^{\mathrm{a}}$ \\
Duration of symptoms & \\
$<1$ month & $32(34.8)$ \\
$>1$ month & $60(65.2)$ \\
Diarrhea & $61(66.3)$ \\
Abdominal pain & $34(37)$ \\
Dyspepsia & $16(17.4)$ \\
Flatulence & $16(17.4)$ \\
NauseaNomiting & $15(16.3)$ \\
Abdominal distension & $13(14.1)$ \\
Pyrosis & $4(4.3)$ \\
Cutaneous manifestations & $9(9.8)$ \\
\hline Exclung symptomatic patients with other possib & \\
\hline
\end{tabular}

${ }^{a}$ Excluding symptomatic patients with other possible explanations
Table 3 Other intestinal parasites observed in stool samples of the study population

\begin{tabular}{ll}
\hline Parasites isolated & $\begin{array}{l}\text { Number of patients (\%) } \\
(n=418)\end{array}$ \\
\hline Pathogenic parasites & $24(5.7)$ \\
Strongyloides stercoralis & $17(4)$ \\
Giardia duodenalis & $10(2.4)$ \\
Hookworms & $8(1.9)$ \\
Trichuris trichiura & $3(0.7)$ \\
Schistosoma mansoni & $2(0.5)$ \\
Schistosoma intercalatum & $2(0.5)$ \\
Hymenolepis nana & $2(0.5)$ \\
Taenia sp. & $1(0.2)$ \\
Ascaris lumbricoides & \\
Parasites of minor importance & $68(16.2)$ \\
Dientamoeba fragilis & $3(0.7)$ \\
Entamoeba histolytica/dispar & \\
Non pathogenic parasites & $66(15.8)$ \\
Endolimax nana & $52(12.4)$ \\
Entamoeba coli & $1(0.2)$ \\
lodamoeba butschlii & $1(0.2)$ \\
Chilomastix mesnili &
\end{tabular}

to treatment varied in this group. Five patients experienced complete resolution of symptoms and 12 reported some clinical improvement but not complete resolution of their illness. Eight patients described no clinical improvement following treatment, and information on the efficacy of treatment was not available for six patients. Therefore, more than $50 \%$ of treated patients reported resolution or improvement of symptoms. Stool examination following treatment was performed for 12 patients. Microbiological cure was observed in seven patients, and five patients still had Blastocystis sp. in their stool. Six patients that experienced microbiological cure were treated with metronidazole, and one had received cotrimoxazole. Four of these patients reported improvement or resolution of symptoms and three patients reported no clinical improvement.

\section{Discussion}

We retrospectively studied 418 adult patients with Blastocystis sp. infection who attended the Vall d'Hebron University Hospital. Most of them were young (median age of 36 years), and foreign-born (> $60 \%$ of patients were born in a country different from Spain). Most of the patients were asymptomatic, but $22 \%$ of them presented with symptoms that were most likely attributable to Blastocystis sp. infection given the absence of any other potential etiology. 
Table 4 Comparison of clinical and epidemiological characteristics between symptomatic and asymptomatic patients with Blastocystis sp. infection attended in Vall d'Hebron University Hospital (2009-2014). Data are reported as number (\%) of patients or median (range)

\begin{tabular}{llll}
\hline & Asymptomatic patients $(n=234)$ & Symptomatic patients ${ }^{\mathrm{a}}(n=92)$ & $P$-value \\
\hline Age, years & $34(18-84)$ & $42.5(18-84)$ & $<0.0001$ \\
Gender, male & $140(59.8 \%)$ & $49(53.3 \%)$ & 0.280 \\
Foreign origin & $210(89.7 \%)$ & $30(32.6 \%)$ & $<0.0001$ \\
Immunosuppressant condition & $47(20.1 \%)$ & $5(5.4 \%)$ & 0.001 \\
\hline
\end{tabular}

${ }^{a}$ Excluding symptomatic patients for other reasons

Interest in Blastocystis sp. has significantly grown in recent years, due to its potential role as a human pathogen [3]. The prevalence of Blastocystis sp. infection varies depending on the study setting, reaching the highest percentages (68-100 \%) among children from developing countries [12, 13]. Few studies have been performed in Spain, and most involve children with prevalences ranging from $5-20 \%[9,10]$. In a previous study carried out by our group among foreign-born HIV infected adult patients, the prevalence of Blastocystis sp. infection was $17.3 \%$ [14].

Seasonal distribution of Blastocystis sp. infection was reported previously in the United Kingdom, with higher incidences observed in summer [15]. In our study, although the number of cases was higher in spring, this was not statistically significant. A previous study performed in the same geographical area (Catalonia, Spain) also showed no seasonal differences [11].

In the present study, the most frequent symptoms observed in patients with illness that could only be attributable to Blastocystis sp. infection were diarrhea, abdominal pain, dyspepsia, flatulence and vomiting, similar to other studies [3]. While its pathogenicity has been questioned, Blastocystis sp. is now thought to cause gastrointestinal symptoms under some circumstances, sometimes of a severe nature [16, 17]. Although less common, cutaneous manifestations have been reported. In some patients acute or chronic urticaria has been observed, unexplained by other causes, with resolution of symptoms described following antiparasitic treatment $[18,19]$. Nine patients in our study presented urticarial dermatitis without a reasonable cause, except for Blastocystis sp. infection. Some suggest that cutaneous symptoms may be caused by disruptions in immune homeostasis as the host produces an inflammatory response against the amoeboid forms of the parasite [20].

It has been suggested that gastrointestinal symptoms could be related with the ST of the parasite, but this remains uncertain [6]. ST-1 seems to be the most pathogenic one, while ST-3, although being the most common ST in humans, is less frequently associated with symptoms $[6,21]$. Parasite loads as observed by light microscopy may also be related to the presence or absence of symptoms, with symptoms occurring more frequently when more than five parasites per highpower field $(400 \times)$ are observed [22].
Blastocystis sp. infection may cause symptoms similar to those attributed to irritable bowel syndrome (IBS). Several studies have shown the possible link between Blastocystis sp. infection and IBS, and Blastocystis sp. infections are more prevalent among patients with IBS when compared to control groups [23,24]. While this is the case, links between IBS and any particular Blastocystis sp. ST have not been observed [25, 26]. A study by Nourrisson et al. revealed that patients with IBS and Blastocystis sp. infection had significantly lower levels of beneficial gut bacteria including Bifidobacterium spp. and Faecalibacterium prausnitzii, supporting that Blastocystis sp. might be linked to the pathophysiology of IBS and intestinal flora imbalance [27]. In our cohort of Blastocystis sp. infected patients, 11 had been diagnosed with IBS, although only one patient received antiparasitic treatment and clinical improvement was not observed. Nevertheless, this association between IBS and Blastocystis sp. requires further investigation given the conflicting reports on this subject [28].

In the present study, the asymptomatic patient group had a higher proportion of foreigners and immunosuppressed patients compared to the symptomatic patient group. These differences could be linked to biases related to the types of patients that are screened for bowel parasites, because fecal microscopic examination for intestinal parasitosis is routinely performed in our hospital on immigrants, particularly those who are immunocompromised regardless of whether they are symptomatic or asymptomatic. Information on the pathogenicity of Blastocystis sp. in immunocompromised patients is unavailable and only limited information on its prevalence in immunosuppressed cohorts has been reported, mostly for HIV infected patients [14, 29, 30].

Only 31 symptomatic patients received antiparasitic treatment for Blastocystis sp. infection in our study, making it difficult to speculate on the usefulness of treatment for Blastocystis sp. infections. Given the controversy surrounding the pathogenic potential of this parasite, physicians are still uncertain as to the value of treating Blastocystis sp. infections. Metronidazole is the most frequently administered drug for Blastocystis sp. infection, though its efficacy is highly variable [3]. Other studies have shown the potential benefits of other drugs, 
such as nitazoxanide, paromomycin or trimethoprimsulfamethoxazole [31, 32]. Despite all the controversies, the treatment success rate achieved in this study (more than $50 \%$ ), suggest that treatment should be considered in patients with chronic symptoms in the absence of other reasonable causes.

This study has some limitations derived from its retrospective nature. Among these limitations is the fact that information retrieved from medical records is not always complete, different physicians were involved in the diagnosis, and differences exist in the way patients were assessed. Diagnosis was based on the microscopic examination of stool samples, lacking information relating to the STs or parasite burden. Moreover, the formalin-ether technique has reduced sensitivity in detecting Blastocystis sp. compared to other techniques which may have reduced its recovery in this cohort [15]. Finally, the small sample size and the varied responses to chemotherapy make it difficult to assess the efficacy of antiprotozoal treatment in this cohort.

\section{Conclusions}

In summary, 418 Blastocystis sp. infected patients were diagnosed in Vall d'Hebron University Hospital over a 5-year period, most of them were foreign-born patients (> $60 \%$ ). Although the vast majority of patients were asymptomatic, $22 \%$ of patients had gastrointestinal symptoms or cutaneous manifestations in the absence of other potential etiologies. Despite the scarcity of information available, and given the safety of antiparasitic treatment, the results of the present study suggest that antiprotozoal treatment should be considered in patients infected with Blastocystis sp. experiencing chronic symptoms. The present study also suggests that Blastocystis sp. are pathogenic at least under some circumstances, and should be considered a cause of illness in the absence of any other etiology.

\section{Abbreviations}

IBS: Irritable bowel syndrome; SSU rRNA: Small subunit rRNA; ST: Subtype; VFR: Visiting friends and relatives

\section{Acknowledgements}

This study was supported by the 6th National Plan (PN) of Research + Development + Innovation (I + D + I) 2008-2011, ISCIII-General Division Networks and Cooperative Research Centres + FEDER funds + Collaborative Research Network on Tropical Diseases (RICET): RD12/0018/0020 and RD12/0018/0011.

\section{Funding}

This research received no specific grant from any funding agency in the public, commercial, or not-for-profit sectors.

\section{Availability of data and materials}

Not applicable.

\section{Authors' contributions}

FS, ASM and IM designed the study, collected and analyzed the data, and wrote the first draft of the paper. ES, CA, JS and IF contributed to data collection, laboratory work and critically reviewed the manuscript for important intellectual content. All authors read and approved the final version of the manuscript.

\section{Competing interests}

The authors declare that they have no competing interests.

\section{Consent for publication}

Not applicable.

Ethics approval and consent to participate

The study protocol was approved by the Ethical Review Board of the Vall d'Hebron University Hospital (Barcelona, Spain). Procedures were performed in accordance with the ethical standards laid down in the Declaration of Helsinki as revised in 2013

\section{Author details}

'Department of Infectious Diseases, Vall d'Hebron University Hospital, Universitat Autònoma de Barcelona, PROSICS Barcelona, Barcelona, Spain. ${ }^{2}$ Department of Microbiology, Vall d'Hebron University Hospital, PROSICS Barcelona, Barcelona, Spain. ${ }^{3}$ Gastroenterology Department, Vall d' Hebron Research Institute, Digestive Diseases Research Unit, Vall d'Hebron University Hospital, Barcelona, Spain. ${ }^{4}$ Department of Medicine, Universitat Autònoma de Barcelona, Centro de Investigación Biomédica en Red de Enfermedades Hepáticas y Digestivas (Ciberehd), Barcelona, Spain. Instituto de Salud Carlos III, National Centre of Microbiology, Madrid, Spain.

Received: 28 June 2016 Accepted: 2 October 2016

Published online: 14 October 2016

\section{References}

1. Fletcher SM, Stark D, Harkness J, Ellis JT. Enteric protozoa in the developed world: a public health perspective. Clin Microbiol Rev. 2012;25:420-49.

2. Stark D, Barratt JLN, van Hal S, Marriott D, Harkness J, Ellis JT. Clinical significance of enteric protozoa in the immunosuppressed human population. Clin Microbiol Rev. 2009;22:634-50.

3. Roberts T, Stark D, Harkness J, Ellis JT. Update on the pathogenic potential and treatment options for Blastocystis sp. Gut Pathog. 2014;6:17.

4. Tan KSW. New insights on classification, identification, and clinical relevance of Blastocystis spp. Clin Microbiol Rev. 2008;21:639-65.

5. Parkar U, Traub RJ, Vitali S, Elliot A, Levecke B, Robertson I, et al. Molecular characterization of Blastocystis isolates from zoo animals and their animalkeepers. Vet Parasitol. 2010;169:8-17.

6. Roberts T, Stark D, Harkness J, Ellis J. Subtype distribution of Blastocystis isolates identified in a Sydney population and pathogenic potential of Blastocystis. Eur J Clin Microbiol Infect Dis. 2013;32:335-43.

7. Bálint A, Dóczi I, Bereczki L, Gyulai R, Szucs M, Farkas K, et al. Do not forget the stool examination! Cutaneous and gastrointestinal manifestations of Blastocystis sp infection. Parasitol Res. 2014;113:1585-90.

8. Leder K, Hellard ME, Sinclair MI, Fairley CK, Wolfe R. No correlation between clinical symptoms and Blastocystis hominis in immunocompetent individuals. J Gastroenterol Hepatol. 2005;20:1390-4.

9. Martín-Sánchez AM, Canut-Blasco A, Rodríguez-Hernández J, MontesMartínez I, García-Rodríguez JA. Epidemiology and clinical significance of Blastocystis hominis in different population groups in Salamanca (Spain). Eur J Epidemiol. 1992;8:553-9.

10. Soriano JM, Domenech G, Martínez MC, Mañes J, Soriano F. Intestinal parasitic infections in hosted Saharawi children. Trop Biomed. 2011;28:557-62.

11. González-Moreno O, Domingo L, Teixidor J, Gracenea M. Prevalence and associated factors of intestinal parasitisation: a cross-sectional study among outpatients with gastrointestinal symptoms in Catalonia. Spain Parasitol Res. 2011;108:87-93.

12. Dib JR, Fernández-Zenoff MV, Oquilla J, Lazarte S, González SN. Prevalence of intestinal parasitic infection among children from a shanty town in Tucuman, Argentina. Trop Biomed. 2015;32:210-5.

13. El Safadi D, Gaayeb L, Meloni D, Cian A, Poirier P, Wawrzyniak I, et al. Children of Senegal River Basin show the highest prevalence of Blastocystis sp ever observed worldwide. BMC Infect Dis. 2014;14:164.

14. Salvador F, Molina I, Sulleiro E, Burgos J, Curran A, Van den Eynde E, et al. Tropical diseases screening in immigrant patients with HIV infection in a European country. Am J Trop Med Hyg. 2013;88:1196-202.

15. Suresh K, Smith $\mathrm{H}$. Comparison of methods for detecting Blastocystis hominis. Eur J Clin Microbiol Infect Dis. 2004;23:509-11. 
16. Andiran N, Acikgoz ZC, Turkay S, Andiran F. Blastocystis hominis, an emerging and imitating cause of acute abdomen in children. J Pediatr Surg. 2006; 41:1489-91.

17. Carrascosa M, Martínez J, Pérez-Castrillón JL. Hemorrhagic proctosigmoiditis and Blastocystis hominis infection. Ann Intern Med. 1996;124:278-9.

18. Gupta R, Parsi K. Chronic urticaria due to Blastocystis hominis. Australas J Dermatol. 2006:47:117-9.

19. Cassano N, Scoppio BM, Loviglio MC, Vena GA. Remission of delayed pressure urticaria after eradication of Blastocystis hominis. Acta Derm Venereol. 2005;85:357-8.

20. Katsarou-Katsari A, Vassalos CM, Tznetou K, Spanakos G, Papadopoulos C Vakalis N. Acute urticaria associated with amoeboid forms of Blastocystis sp subtype 3. Acta Derm Venereol. 2008:88:80-1.

21. Yan Y, Su S, Lai R, Liao H, Ye J, Li X, et al. Genetic variability of Blastocystis hominis isolates in China. Parasitol Res. 2006:99:597-601.

22. Kaya S, Cetin ES, Aridogan BC, Arikan S, Demirci M. Pathogenicity of Blastocystis hominis, a clinical reevaluation. Turkiye Parazitol Derg. 2007:31:184-7.

23. Jiménez-González DE, Martínez-Flores WA, Reyes-Gordillo J, Ramírez-Miranda ME, Arroyo-Escalante S, Romero-Valdovinos $\mathrm{M}$, et al. Blastocystis infection is associated with irritable bowel síndrome in a Mexican patient population. Parasitol Res. 2012;110:1269-75.

24. Yakoob J, Jafri W, Jafri N, Khan R, Islam M, Beg MA, Zaman V. Irritable bowel syndrome: in search of an etiology: role of Blastocystis hominis. Am J Trop Med Hyg. 2004;70:383-5.

25. Das R, Khalil S, Mirdha BR, Makharia GK, Dattagupta S, Chaudhry R. Molecular characterization and subtyping of Blastocystis species in irritable bowel syndrome patients from North India. PLoS One. 2016;11, e0147055.

26. Mattiucci S, Crisafi B, Gabrielli S, Paoletti M, Cancrini G. Molecular epidemiology and genetic diversity of Blastocystis infection in humans in Italy. Epidemiol Infect. 2016;144:635-46.

27. Nourrisson C, Scanzi J, Pereira B, NkoudMongo C, Wawrzyniak I, Cian A, et al. Blastocystis is associated with decreased of fecal microbiota protective bacteria: comparative analysis between patients with irritable bowel syndrome and control subjects. PLoS One. 2014;9, e111868.

28. Krogsgaard LR, Engsbro AL, Stensvold CR, Nielsen HV, Bytzer P. The prevalence of intestinal parasites is not greater among individuals with irritable bowel syndrome: a population-based case-control study. Clin Gastroenterol Hepatol. 2015;13:507-13.

29. Tian LG, Wang TP, Lv S, Wang FF, Guo J, Yin XM, et al. HIV and intestinal parasite co-infections among a Chinese population: an immunological profile. Infect Dis Poverty. 2013;2:18.

30. Tan TC, Ong SC, Suresh KG. Genetic variability of Blastocystis sp isolates obtained from cancer and HIV/AIDS patients. Parasitol Res. 2009;105:1283-6.

31. Cimerman S, Ladeira MC, Iuliano WA. Blastocystis: nitazoxanide as a new therapeutic option. Rev Soc Bras Med Trop. 2003;36:415-7.

32. Ok UZ, Girginkardesler N, Balcioglu C, Ertan P, Pirildar T, Kilimcioglu AA. Effect of trimethoprim-sulfamethoxazole in Blastocystis hominis infection. Am J Gastroenterol. 1999;94:3245-7.

\section{Submit your next manuscript to BioMed Central and we will help you at every step:}

- We accept pre-submission inquiries

- Our selector tool helps you to find the most relevant journal

- We provide round the clock customer support

- Convenient online submission

- Thorough peer review

- Inclusion in PubMed and all major indexing services

- Maximum visibility for your research

Submit your manuscript at www.biomedcentral.com/submit

CBiomed Central 